\title{
ON DAHLBERG'S LUSIN AREA INTEGRAL THEOREM
}

\author{
MARIUS MITREA
}

(Communicated by J. Marshall Ash)

\begin{abstract}
We give new proofs to the Lusin area integral theorem of Dahlberg. Our techniques rely on the theory of elliptic boundary value problems on nonsmooth domains and are shown to extend to other important cases, including systems of equations.
\end{abstract}

\section{INTRODUCTION}

The graph version of the Lusin area integral theorem of Dahlberg [D1] asserts that for any harmonic function $u$ vanishing at infinity in $\Omega$ one has

$$
\int_{\partial \Omega}|u(X)|^{2} d S(X) \leq C \iint_{\Omega}|\nabla u(X)|^{2} \delta(X) d X
$$

and

$$
\iint_{\Omega}|\nabla u(X)|^{2} \delta(X) d X \leq C \int_{\partial \Omega}|u(X)|^{2} d S(X)
$$

Here $\Omega$ is an unbounded Lipschitz domain in $\mathbb{R}^{n+1}, \delta(X):=\operatorname{dist}(X, \partial \Omega)$, $d S$ is the surface area on $\partial \Omega$, and all constants depend solely on the Lipschitz character of $\partial \Omega$. In fact, (1) and (2) also hold true in the case in which $\Omega$ is a bounded Lipschitz domain, provided $u$ is normalized by $u(P)=0$, for some fixed $P \in \Omega$.

Inequalities of this type have a long history going back (at least for the upper half space) to the work of Calderón [Ca], Segovia [S], Burkholder and Gundy [BG], Fefferman and Stein [FS]. Actually, if $\Omega$ is a bounded sufficiently smooth domain, then so is $\delta$, and then (1) and (2) follow by simple integrations by parts (see, e.g., p. 279 in [Ne]). Without attempting to give a complete account of work related to this subject, we will just mention that in the case of an unbounded Lipschitz domain in $\mathbb{C}$ inequalities of this type have been also obtained in [K1] by means of complex variable techniques.

Such quadratic estimates turned out to be essential tools for proving the boundedness of the Cauchy integral and other related operators on Lipschitz curves in $\mathbb{C}[\mathrm{CJS}]$ and even Lipschitz hypersurfaces in $\mathbb{R}^{n+1}$ [LMS]. They were also used in connection with elliptic boundary value problems on nonsmooth

Received by the editors August 9, 1993.

1991 Mathematics Subject Classification. Primary 42B20, 31B10; Secondary 31B35.

The author was partially supported by ONR Grant N0001-90-J-1343. 
domains [Ve],[DKV1]. An important feature of these inequalities resides in the fact that they could be used to prove Sobolev-Besov space regularity results for the solutions of boundary value problems for the Laplacian [Fa],[JK]. Furthermore, these estimates can be also interpreted as a higher-dimensional version of the Burkholder-Gundy-Silverstein theorem [BGS] on nonsmooth domains. Indeed, they readily imply that $\left\|u^{*}\right\|_{L^{2}(\partial \Omega)} \approx \sum_{j}\left\|u_{j}^{*}\right\|_{L^{2}(\partial \Omega)}$, where $\left\{u_{j}\right\}_{j}$ are the harmonic conjugates of $u$ (as usual, * stands for the usual nontangential maximal operator); cf. also [Ve].

The aim of this paper is to present several new proofs and extensions of the above estimates. An interesting feature is that, in some sense, (1) and (2) are dual to each other so that it actually suffices to dispose of only one of them. This can be done, for instance, by an application of (a simple) form of the $T(b)$ theorem [MM],[DJS],[Se]. The techniques involved heavily depend on results from the theory of elliptic PDE's on nonsmooth domains [Ve],[DK], and are also shown to work in several other instances, including even systems of equations (cf. $\S 6)$.

\section{Proof of (2) $\Rightarrow(1)$}

Let $n(X)$ be the outward unit normal defined at a.e. $X \in \partial \Omega$, and let $\sigma_{n}$ be the area of the unit sphere in $\mathbb{R}^{n+1}$. Consider

$$
\mathscr{D} f(X):=\frac{1}{\sigma_{n}} \int_{\partial \Omega} \frac{\langle n(Y), X-Y\rangle}{|X-Y|^{n+1}} f(Y) d S(Y), \quad X \notin \partial \Omega,
$$

the usual double layer potential operator on $\partial \Omega$, and let

$$
\left( \pm \frac{1}{2}+\mathscr{K}\right) f(X):=\lim _{\substack{Y \rightarrow X \\ Y \in \pm+X}} \mathscr{D} f(Y), \quad \text { at a.e. } X \in \partial \Omega,
$$

be its nontangential boundary traces. Here, $\gamma$ is an upright circular cone, which is sufficiently sharp and centered at the origin of $\mathbb{R}^{n+1}$ (cf. [Ve],[DK]).

For a harmonic function $u$ in $\Omega$ which is small at infinity and $s \geq 0$, Verchota's representation theorem [Ve] for $v_{s}:=\left.\left(\partial_{0} u\right)\right|_{\Omega+(s, 0)}$ gives that, for any $t \geq 0$,

$$
\begin{aligned}
&\left(\partial_{0} u\right)(X+(s+t, 0)) \\
&=\frac{1}{\sigma_{n}} \int_{\partial \Omega} \frac{\langle n(Y), X-Y+(t, 0)\rangle}{|X-Y+(t, 0)|^{n+1}}\left[\left(\frac{1}{2}+\mathscr{K}_{s}\right)^{-1} v_{s}\right](Y+(s, 0)) d S(Y),
\end{aligned}
$$

where $\mathscr{K}_{s}$ stands for the singular double layer potential operator corresponding to the hypersurface $\partial \Omega+(s, 0)$. Now let $h$ be an arbitrary function in $L_{\text {comp }}^{\infty}(\partial \Omega)$. Differentiating the above equality with respect to $t$, making then $s=t$, and finally integrating against $\int_{\partial \Omega} \int_{0}^{\infty} h(X) t d t d S(X)$, we get

$$
\begin{aligned}
\int_{0}^{\infty} \int_{\partial \Omega}\left(\partial_{0}^{2} u\right)(X+(2 t, 0)) h(X) t d t d S(X) \\
\quad=\frac{1}{\sigma_{n}} \int_{0}^{\infty} \int_{\partial \Omega} \partial_{0}(\mathscr{D} h)(Y-(t, 0))\left[\left(\frac{1}{2}+\mathscr{K}_{t}\right)^{-1} v_{t}\right](Y+(t, 0)) t d t d S(Y) .
\end{aligned}
$$


In the left-hand side of this identity we integrate by parts twice in the variable $t$ and, as $u$ vanishes at infinity, obtain

$$
\text { the left-hand side }=\frac{1}{4} \int_{\partial \Omega} u(X) h(X) d S(X) .
$$

As for the right-hand side, we first use the Schwarz inequality in the variable $Y$, remove $\left(1 / 2+\mathscr{K}_{t}\right)^{-1}$ (as this is an isomorphism of $L^{2}(\partial \Omega+(t, 0))$ with norm uniformly bounded in $t[\mathrm{Ve}],[\mathrm{DK}])$, then use once again Schwarz inequality for the measure $t d t$, and finally end up with

the right-hand side

$$
\leq C\left(\iint_{\Omega}|\nabla u(Y)|^{2} \delta(Y) d Y\right)^{1 / 2}\left(\iint_{\Omega}|\nabla \mathscr{D} h(Y)|^{2} \delta(Y) d Y\right)^{1 / 2} .
$$

This together with (3), (2) and the usual argument completes the proof of the implication $(2) \Rightarrow(1)$.

\section{Proof of (1) $\Rightarrow(2)$}

Here we closely follow the lines of [CJS]. Let $\Omega_{+}:=\Omega, \Omega_{-}:=\mathbb{R}^{n+1} \backslash \bar{\Omega}$, and

$$
\mathscr{H}_{ \pm}:=\left\{F: \Omega_{ \pm} \rightarrow \mathbb{C} ;\|F\|_{\mathscr{H}_{ \pm}}:=\left(\iint_{\Omega_{ \pm}}|F(X)|^{2} \delta(X) d X\right)^{1 / 2}<+\infty\right\} .
$$

Consider the operator $\mathscr{T}$ acting on functions defined in $\Omega$ by

$$
\mathscr{T} F(X):=\iint_{\Omega}\left(\nabla_{I} K\right)(Y, X) F(Y) \delta(Y) d Y, \quad X \in \partial \Omega,
$$

where $K(X, Y)$ is the kernel of the singular double layer potential operator $\mathscr{K}$. By the Schur test, $\nabla \mathscr{T}: \mathscr{H}_{+} \rightarrow \mathscr{H}_{-}$is readily seen to be bounded so that, by (1), $\mathscr{T}: \mathscr{H}_{+} \rightarrow L^{2}(\partial \Omega)$ is bounded too.

Now let $u=\mathscr{D} f$, for some $f \in L^{2}(\partial \Omega)$ so that $\left\|u^{*}\right\|_{L^{2}(\partial \Omega)} \approx\|f\|_{L^{2}(\partial \Omega)}$ ([Ve]). Since for any $F \in \mathscr{H}_{+}$,

$$
\iint_{\Omega} \nabla(\mathscr{D} f)(X) F(X) \delta(X) d X=\int_{\partial \Omega} f(Y)(\mathscr{T} F)(Y) d S(Y),
$$

a simple duality argument completes the proof of the implication $(1) \Rightarrow(2)$.

\section{A DIRECT PROOF OF (2)}

In this section we present several ways of directly disposing of (2). For instance, if $g$ is the usual Littlewood-Paley function (see, e.g., [St]) and $\mathscr{C}$ is the higher-dimensional Cauchy integral operator (cf., e.g., [Me],[Mu],[Mc]), then for any $u=\mathscr{D} f$ in $\Omega$, with $f \in L^{2}(\partial \Omega)$, we have

$$
\begin{aligned}
\left(\iint_{\Omega}|\nabla u|^{2} \delta\right)^{1 / 2} & \leq C\|g(\mathscr{D} f)\|_{L^{2}(\partial \Omega)} \leq C\|g(\mathscr{C} f)\|_{L^{2}(\partial \Omega)} \\
& \leq C\|f\|_{L^{2}(\partial \Omega)} \approx\|u\|_{L^{2}(\partial \Omega)} .
\end{aligned}
$$

In fact, it is possible to prove that all the above quantities are equivalent and hence obtain a direct proof of (1) and (2); see [M1-2] for such an approach. 
However, the second inequality is a mere consequence of the fact that $\mathscr{D} f=$ $\operatorname{Re}(\mathscr{C} f)$ (cf., e.g., [Mc]).

As for the third inequality, one can use the "analyticity" of $\mathscr{C} f$ to check the hypotheses of (an easy version of) the $T(b)$ theorem from which this follows (cf. [Me] in the two-dimensional case and [M1] in arbitrary dimensions). See also [K1] for a direct proof in the complex plane case. Actually, one can prove directly that $\|g(\mathscr{D} f)\|_{L^{2}(\partial \Omega)} \leq C\|f\|_{L^{2}(\partial \Omega)}$ by relying on a version of the $\mathrm{T}(\mathbf{b})$ theorem for singular integrals with harmonic kernels (cf., e.g., [Ta]).

Another way of justifying the estimate $\|g(\mathscr{C} f)\|_{L^{2}(\partial \Omega)} \leq C\|f\|_{L^{2}(\partial \Omega)}$, which also has the advantage of generalizing to other situations, is to use Semmes's square function theorem [Se]. Consider $\varphi: \mathbb{R}^{n} \rightarrow \mathbb{R}$ the Lipschitz function whose graph is $\partial \Omega$. Let $\theta_{t}(x, y):=t\left(\nabla^{2} \Gamma\right)(\varphi(x)-\varphi(y)+t, x-y)$, for $t>0$, $x, y \in \mathbb{R}^{n}$, and $\Gamma(X):=-(n-1)^{-1} \sigma_{n}^{-1}|X|^{1-n}$, the canonical fundamental solution for the Laplacian in $\mathbb{R}^{n+1}(n \geq 2)$. Also set

$$
\boldsymbol{\Theta}_{t} f(x):=\int_{\mathbb{R}^{n}} \theta_{t}(x, y) f(y) d y, \quad x \in \mathbb{R}^{n}, \quad t>0 .
$$

In this context, Semmes's theorem asserts that if there exists a para-accretive function $b \in L^{\infty}\left(\mathbb{R}^{n}\right)$ (cf., e.g., [Se]) such that

$$
\int_{\mathbb{R}^{n}} \theta_{t}(x, y) b(y) d y=0, \quad x \in \mathbb{R}^{n}, \quad t>0,
$$

then

$$
\int_{0}^{\infty}\left\|\boldsymbol{\Theta}_{t} f\right\|_{L^{2}\left(\mathbb{R}^{n}\right)}^{2} \frac{d t}{t} \leq C\|f\|_{L^{2}(\partial \Omega)}^{2} .
$$

This clearly furnishes the desired estimate, as the left-hand side of (5) is essentially $\|g(\mathscr{D} f)\|_{L^{2}(\partial \Omega)}^{2}$. Finally, to see that (4) is fulfilled, it suffices to note that if $K(X, Y)$ is as in $\S 3$, then

$$
\int_{\partial \Omega} \nabla K(X, Y) d S(Y)=0, \quad \text { for all } X \in \Omega,
$$

as one can easily check (for instance by using Green's theorem) that $\nabla \mathscr{D}(1)=$ 0 .

\section{A DIRECT PROOF OF (1)}

Perhaps the easiest way of proving (1) directly is by using Dahlberg's estimate [D2]:

$$
\left|\iint_{\Omega}\langle\nabla u, A\rangle d X\right| \leq C\|g(u)\|_{L^{2}(\partial \Omega)}\left(\|g(A)\|_{L^{2}(\partial \Omega)}+\left\|A^{*}\right\|_{L^{2}(\partial \Omega)}\right) .
$$

Here, $u$ is a harmonic function in $\Omega$ and $A$ is a smooth vector field in $\Omega$, small at infinity. It is easy to see that (6) implies (1), as we can write

$$
\int_{\partial \Omega}|u|^{2} d S \leq C\left|\iint_{\Omega} \partial_{0} u u d X\right|=\left|\iint_{\Omega}\langle\nabla u,(u, 0, \ldots, 0)\rangle d X\right| .
$$

The rest of this section is devoted to presenting an elementary, simple proof of (6), along the lines of [D2], [LMS], [K2]. 
Let $\varphi: \mathbb{R}^{n} \rightarrow \mathbb{R}$ be the Lipschitz continuous function whose graph is $\partial \Omega$. Choose $\theta$ a positive, radial, smooth, compactly supported function on $\mathbb{R}^{n}$, with integral one, and let

$$
\varphi(t, x):=\lambda t+\left(\theta_{t} * \varphi\right)(x), \quad(t, x) \in \mathbb{R}_{+}^{n+1},
$$

be the Kenig-Stein function. For a sufficiently large constant $\lambda$, this function has a bounded gradient, $\partial_{t} \varphi(t, x) \geq 1$, and $\left|\nabla^{2} \varphi(t, x)\right|^{2} t d t d x$ is a Carleson measure on $\mathbb{R}_{+}^{n+1}$.

Next, we shall make the change of variable $X=\varphi(t, x)$, so that we are led to considering terms of the form

$$
\int_{0}^{\infty} \int_{\mathbb{R}^{n}} b_{j}(t, x) \partial_{j} u(t, x) A_{j}(t, x) d t d x,
$$

with $b \in L^{\infty}$ and $|\nabla b(t, x)|^{2} t d t d x$ Carleson measure. Since eventually passing from $u$ to one of its harmonic conjugates leaves the right-hand side of (6) invariant, we may assume that $\partial_{j} u(t, x)$ appears above only for $j \neq 0$. Writing $1=\frac{d}{d t} t$ under the integral sign and integrating by parts, we obtain terms of the form $\iint t b \partial_{x} u \partial_{t} A d t d x$ or $\iint t \partial_{t} b \partial_{x} u A d t d x$ (with $\partial_{t}$ and $\partial_{x}$ eventually interchanged).

Finally, by the Schwarz inequality and the usual Carleson measure type estimate, we can obviously dominate these integrals with bounds of the right order.

\section{OTHER APPLICATIONS}

Here we shall briefly outline further extensions and applications of the techniques presented in the previous sections.

Our first remark concerns the so called metaharmonic functions, i.e., functions annihilated by the Helmholtz operator $\Delta+z$, with $z$ a complex number, $z \notin \mathbb{R}_{+}$. An acoustic potential theory on nonsmooth domains, parallel to the one for the Newtonian potential as presented in [Ve],[DK], is available from [BS],[M3],[TW]. Actually, all we need here are the corresponding Rellich type identities for the graph case, which can easily be deduced as in e.g. [ $\mathrm{Br}, \mathrm{p} .355$ ]. With this at hand, the reasonings from the sections 2 and 4 get through, and the conclusion is that (1) and (2) are also valid for metaharmonic functions. Implications to the regularity of the solutions of boundary value problems for the Helmholtz operator in Lipschitz domains are examined to a certain extent in [M3] and [MTW].

Another example is furnished by the solutions to the Lamé system of equations

$$
\mu \triangle \vec{u}+(\lambda+\mu) \nabla \operatorname{div} \vec{u}=0,
$$

with $\lambda>0, \mu \geq 0$. A complete $L^{2}$-theory in bounded Lipschitz domains is presented in [DKV2]. However, it can easily be checked that the results carry over the case of unbounded Lipschitz domains too. Therefore, as before, the estimates (1) and (2) can be shown to be valid in this case also.

Finally, we remark that, with natural modifications, all these results continue to hold in the bounded Lipschitz domain case. This can be seen by a localization argument similar to the one presented for instance in [DK]. 


\section{REFERENCES}

[Br] R. Brown, The method of layer potentials for the heat equation in Lipschitz cylinders, Amer. J. Math. 111 (1989), 339-379.

[BS] R. Brown and Z. Shen, The initial-Dirichlet problem for a fourth-order parabolic equation in Lipschitz cylinders, Indiana Univ. Math. J. 39 (1990), 1313-1353.

[BG] D. L. Burkholder and R. F. Gundy, Distribution function inequalities for the area integral, Studia Math. 44 (1972), 527-544.

[BGS] D. L. Burkholder, R. F. Gundy, and M. L. Silverstein, A maximal function characterization of the class $H^{p}$, Trans. Amer. Math. Soc. 157 (1971), 137-153.

[Ca] A. P. Calderón, Commutators of singular integral operators, Proc. Nat. Acad. Sci. U.S.A. 53 (1965), 1092-1099.

[CJS] R. R. Coifman, P. Jones, and S. Semme, Two elementary proofs of the $L^{2}$ boundedness of the Cauchy integrals on Lipschitz curves, J. Amer. Math. Soc. 2 (1989), 553-564.

[D1] B. E. J. Dahlberg, Weighted norm inequalities for the Lusin area integral and the nontangential maximal function for functions harmonic in a Lipschitz domain, Studia Math. 67 (1980), 297-314.

[D2] _ Poisson semigroups and singular integrals, Proc. Amer. Math. Soc. 97 (1986), 41-48.

[DK] B. E. J. Dahlberg and C. E. Kenig, Hardy spaces and the $L^{p}-$ Neumann problem for Laplace's equation in a Lipschitz domain, Ann. of Math. (2) 125 (1987), 437-465.

[DKV1] B. E. J. Dahlberg, C. E. Kenig, and G. Verchota, The Dirichlet problem for the biharmonic equation in a Lipschitz domain, Ann. Inst. Fourier (Grenoble) 36 (1986), 109-135.

[DKV2] __ Boundary value problems for the systems of elastostatics in Lipschitz domains, Duke Math. J. 57 (1988), 795-818.

[DJS] G. David, J.-L. Journé, and S. Semmes, Operatéurs de Calderó-Zygmund, fonctiones paraaccretives et interpolation, Rev. Mat. Iberoamericana 1 (1985), 1-56.

[Fa] E. Fabes, Layer potential methods for boundary value problems on Lipschitz domains, Potential Theory, Surveys and Problems (J. Král et al., eds.), Lecture Notes in Math., vol. 1344, Springer-Verlag, New York, 1988, pp. 55-80.

[FS] C. Fefferman and E. M. Stein, $H^{p}$ spaces of several variables, Acta Math. 129 (1972), 137-193.

[JK] D. S. Jerison and C. E. Kenig, Boundary value problems on Lipschitz domains, Studies in Partial Differential Equations (W. Littman, ed.), Stud. Math., vol. 23, Math. Assoc. America, Washington, D. C., 1982, pp. 1-68.

[K1] C. E. Kenig, Weighted $H^{p}$ spaces on Lipschitz domains, Amer. J. Math. 102 (1980), 129-163.

[K2] _ Square function estimates for solutions to elliptic equations and systems on Lipschitz domain, Proc. of the 17th Spring Lecture Series in Mathematics (Fayetteville, 1993) (to appear).

[LMS] C. Li, A. McIntosh, and S. Semmes, Convolution singular integrals on Lipschitz surfaces, J. Amer. Math. Soc. 5 (1992), 455-481.

[Mc] A. McIntosh, Clifford algebras and the higher dimensional Cauchy integral, Approximation Theory and Function Spaces, Banach Center Publ., vol. 22, PWN, Warsaw, 1989, pp. 253-267.

[MM] A. McIntosh and Y. Meyer, Algebres d'opérateurs definis par des integrales singulieres, C. R. Acad. Sci. Paris Sér. I Math. 301 (1985), 395-397.

[Me] Y. Meyer, Ondelettes et opérateurs, Hermann, Paris, 1990.

[M1] M. Mitrea, Singular integrals, Hardy spaces and Clifford wavelets, Lecture Notes in Math., vol. 1575, Springer-Verlag, Berlin and New York, 1994.

[M2] _ Clifford algebras and boundary estimates for harmonic functions, Proc. of the Third Internat. Conf. on "Clifford Algebras and Their Applications in Mathematical Physics", Kluwer Acad. Publ., New York, 1993.

[M3] _ Boundary value problems and Hardy spaces associated to the Helmholtz equation on Lipschitz domains, submitted. 
[MTW] M. Mitrea, R. Torres, and G. Welland, Layer potential techniques in electromagnetism on $C^{1}$ and Lipschitz domains, preprint.

[Mu] M. A. M. Murray, The Cauchy integral, Calderón commutators and conjugations of singular integrals in $\mathbb{R}^{m}$, Trans. Amer. Math. Soc. 289 (1985), 497-518.

[Ne] J. Nečas, Les méthodes directes en théorie des équations élliptique, Academia, Prague, 1967.

[S] C. Segovia, On the area function of Lusin, Studia Math. 33 (1969), 312-343.

[Se] S. Semmes, Square function estimates and the $T(b)$ theorem, Proc. Amer. Math. Soc. 110 (1990), 721-726.

[St] E. M. Stein, Singular integrals and differentiability properties of functions, Princeton Univ. Press, Princeton, NJ, 1970.

[Ta] T. Tao, Convolution operators generated by right-monogenic and harmonic kernels, preprint.

[TW] R. Torres and G. Welland, The Helmholtz equation and transmission problems with Lipschitz interfaces, preprint.

[Ve] G. Verchota, Layer potentials and regularity for the Dirichlet problem for Laplace's equation in Lipschitz domains, J. Funct. Anal. 59 (1984), 572-611.

Institute of Mathematics of the Romanian ACademy, P.O. Box 1-764, RO-70700 BuchAREST, ROMANIA

Current address: School of Mathematics, University of Minnesota, 127 Vincent Hall, 206 Church

St. SE, Minneapolis, Minnesota 55455

E-mail address: mitrea@math.umn.edu 